\title{
Actionneurs linéaires : MRVlin et MSlin, un projet pédagogique
}

\author{
Lotfi BAGHLI $^{1}$, Abderrezak REZZOUG ${ }^{2}$ \\ ${ }^{1}$ IUFM de Lorraine, 5 rue Paul Richard, C.O. N³, 54528 Laxou Cedex, Lotfi.Baghli@green.uhp-nancy.fr \\ ${ }^{2}$ Université Henri Poincaré, Faculté des Sciences, BP 239, 54500 Vandoeuvre, Abderrezak.Rezzoug@green.uhp- \\ nancy.fr
}

\begin{abstract}
RESUME Cet article fait le point sur une expérience que l'on mène à la Faculté des Sciences et Techniques, Université Henri Poincaré (UHP), autour d'un nouveau module d'enseignement sur les actionneurs linéaires en Master $1^{\text {ère }}$ année et pour lequel nous avons construit des machines linéaires de dimensions réduites mues par une électronique de puissance et de commande embarquées. Un logiciel de simulation avec une représentation et un rendu 3D temps réel a également été réalisé. Les programmes de commande et les schémas électroniques sont en libre téléchargement ainsi que les animations et les vidéos réalisées.
\end{abstract}

Mots clés : Actionneur linaire, moteur synchrone, moteur à réluctance variable, dsPIC, microcontrôleur, C++, OpenGL.

\section{INTRODUCTION}

L'idée de ce projet est liée à un nouveau cours mis en place lors du passage au système LMD et tient compte du fait que beaucoup de nos étudiants pensent déjà tout connaître des machines électriques qu'ils ont vu, pour la plupart, au moins une fois dans leur cursus. Nous avons proposé en M1 un cours sur les actionneurs linéaires et nous avions besoin de maquettes de Travaux Pratiques et de nouveaux démonstrateurs.

Nous nous sommes fixés le cahier des charges suivant : les machines doivent être de taille réduite (une dizaine de centimètres) de manière à pouvoir les utiliser dans une salle de cours ou sur une paillasse de TP et pour être facilement transportables pour des manifestations scientifiques (Fête de la Science, journée portes ouvertes...). Elles doivent être alimentées sous $15 \mathrm{~V}$ continu et 2,5 A au maximum.

Elles doivent avoir une structure "ouverte" pour que l'on puisse voir leur constitution.

L'électronique de puissance (onduleur triphasé) et l'électronique de commande (microcontrôleur) embarquées doivent être de même dimension que le moteur. Le tout doit être facile à construire et bon marché, de manière à permettre aux étudiants ou aux collègues de les dupliquer.

On a voulu réaliser trois démonstrateurs [1] : Machine à Réluctance Variable linéaire (MRVlin), Machine Asynchrone linaire (MASlin) et Machine Synchrone linéaire (MSlin). La MASlin n'a pas pu être réalisée pour un certains nombre de raisons que l'on explicitera. En amont de la réalisation expérimentale, nous avons élaboré des modèles numériques des machines linéaires (MRV, MS) ainsi que leur contrôle et nous les avons incorporés à un logiciel de simulation et de rendu 3D que vous avions réalisé au préalable en C++ / OpenGL [2]. Il nous a permis de représenter et de vérifier nos choix et d'illustrer les cours, TD et TP avec des représentations des machines et des configurations à un instant donné (courants dans les enroulements, état des sondes à effet Hall, séquences de commande, valeur des force Fx, Fy, Fz,_Fig 2 à Fig 4_).

\section{CHOIX TECHNOLOGIQUE ET DIMENSIONNEMENT}

Le cahier des charges que nous nous sommes fixé prévoyait de faibles dimensions afin de rendre les machines facilement manipulables et transportables.

Le niveau de tension $(<15 \mathrm{~V})$ permettrait à l'actionneur de pouvoir être alimenté par une alimentation standard de table de salle de TP (30V / 2.5A). Ce niveau de tension offre aussi l'avantage de ne pas poser de problème de sécurité et d'isolation particulier et ainsi permettre aux étudiants de mettre les doigts sur la machine et sur la carte de commande, d'aller observer, à l'oscilloscope, les signaux de commande et de puissance.

Contrairement aux moteurs ronds qui sont fermés, ces machines linéaires permettent une vue "écorchée" réelle et en fonctionnement de la machine.

Certes, le rendement et les forces ne seront pas optimisés mais ils doivent rester suffisants pour montrer les phénomènes électromagnétiques mis en jeu.

Le courant pose une contrainte d'échauffement, de dimension des conducteurs des bobines et donc de taille des encoches. L'entrefer, pose celle de la réalisation mécanique et a un impact considérable sur les forces résultantes, il fallait donc une réalisation suffisamment précise.

Nous avons choisi comme matériau ferromagnétique une barre en acier standard (6m x $30 \mathrm{~mm}$ x $15 \mathrm{~mm}$ ) qui, usinée, donnerait les mobiles et les rails.

\subsection{Microcontrôleur}

Pour pouvoir contrôler les courants, il faut un convertisseur statique piloté par une commande à Modulation de Largeur d'Impulsion (MLI ou PWM). Le choix d'un microcontrôleur disposant de sorties MLI s'est imposé. Il est associé à un onduleur de tension triphasé. Nous avons retenu le L6234 de STMicroelectronics disponible sur le marché pour une douzaine d'euros et qui peut délivrer 2,5 A dans sa 
version DIP (20 broches). Sa taille, incroyablement petite, convient tout à fait à l'application. Pour le microcontrôleur, nous cherchions un microcontrôleur bon marché, souple d'utilisation, disposant d'une interface de débogage et qui se programme en C.

L'intérêt du langage C, par rapport à l'assembleur, réside dans une meilleure prise en main par les étudiants et une facilité de portage vers d'autres platesformes. De plus, vu la puissance des microcontrôleurs actuels et de la qualité des compilateurs, et concernant ces applications, nous pensons que la recherche d'une minimisation de temps de calcul des routines, par l'assembleur, ne se justifie plus.

Notre choix s'est porté vers un microcontrôleur 16 bits de Microchip, dont un collègue rencontré lors du CETSIS'2005 à Nancy nous avait vanté les mérites. Ce constructeur, fort célèbre pour son PIC (microcontrôleur 8 bits), a sorti une version 16 bits très simple d'implantation mais qui offre jusqu'à 30 MIPS (Millions d'Instructions Par Seconde).

Ces composants sont appelés DSC (Digital Signal Controller) puisqu'ils intègrent des éléments d'un DSP mais se rapprochent plus du microcontrôleur.

Nous avons adopté le dsPIC $30 F 3010$ pour ce projet. Il existe en version PDIP 28 broches donc facilement "soudable". Outre qu'il offre la possibilité de se programmer (flasher) in-situ, sur le circuit imprimé final, il dispose d'un convertisseur analogiquenumérique (ADC) 10 bits, de nombreuses broches d'entrées/sortie (E/S), d'un module PWM, d'une interface pour codeur incrémental (QEP), d'une liaison série asynchrone (UART), de liaisons séries synchrones (SPI, $\left.\mathrm{I}^{2} \mathrm{C}\right)$...
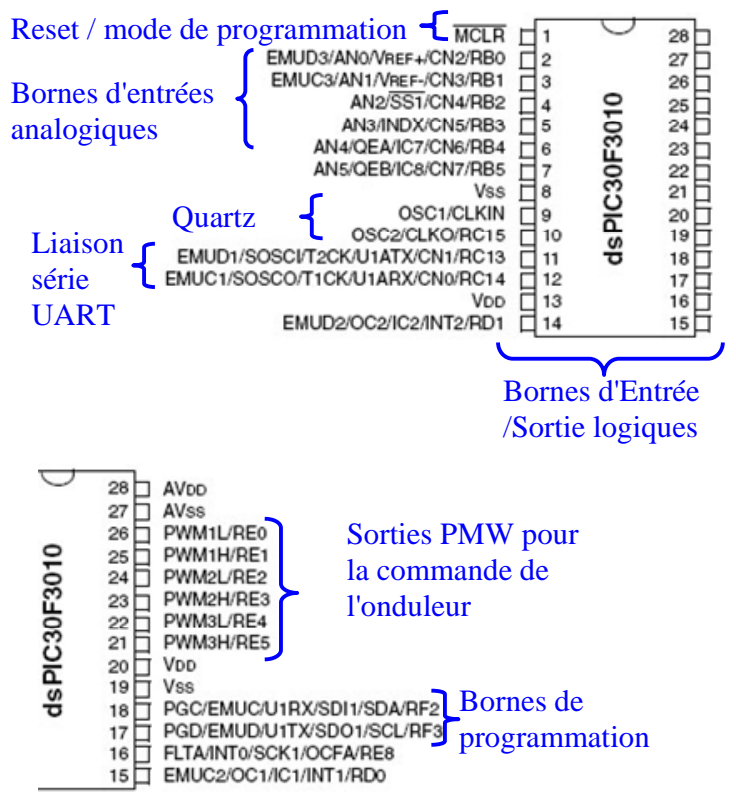

Fig 1. Brochage du dsPIC 30F3010.
Nous avons alimenté le dsPIC sous 5V ce qui permet d'atteindre 20 MIPS voire même 30 MIPS avec un quartz de 7,3728 MHz. On peut alors opérer avec des fréquences de MLI de 10 à $16 \mathrm{kHz}$ et des périodes d'échantillonnage réduites. Le contrôle peut ainsi répondre très rapidement et garantir un parfait lissage du courant du moteur linéaire.

La sortie UART TX a été connectée via un MAX232 à l'entrée RS232 RX d'un PC équipé du Terminal Windows. On peut donc observer les valeurs des variables internes du programme. Ceci est très utile pour le débogage du programme en cours de développement ou pour la supervision de processus à distance.

Ce microcontrôleur s'est révélé très simple d'utilisation et rapide à mettre en œuvre. Nous l'utilisons actuellement dans d'autres projets de Master1 et pour développer d'autres maquettes pédagogiques (sustentation magnétique, horloge à persistance rétinienne, commande $\mathrm{V} / \mathrm{f}$, affichage sur LCD graphique) [1].

\subsection{Simulation}

Pour la simulation, nous avons développé un logiciel en $\mathrm{C}++$ (sous Borland C++ Builder 6) doté d'une interface OpenGL qui permet d'animer des petits robots à 2 ou 4 roues soit en pilotage manuel, soit en automatique avec gestion des collisions [2]. Nous avons intégré tout naturellement nos actionneurs linéaires dans cet environnement de simulation.

Les modèles des moteurs doivent être simples (calcul des forces mises en jeu, intégration numérique des équations différentielles) mais suffisamment fiables pour représenter le comportement des actionneurs réels et ainsi aider à la compréhension de leur fonctionnement (Fig 2 à Fig 4).

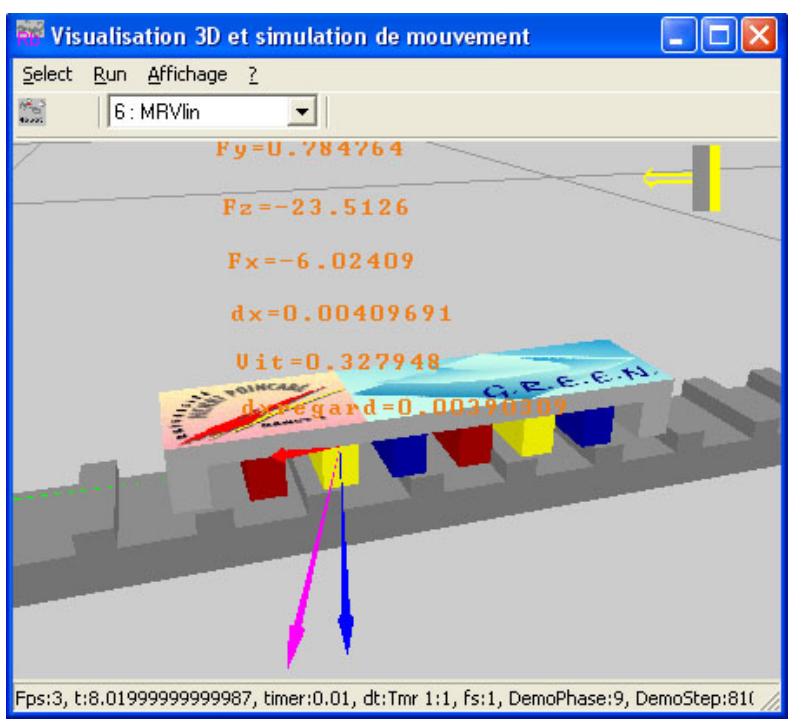

Fig 2. MRVlin en simulation. 


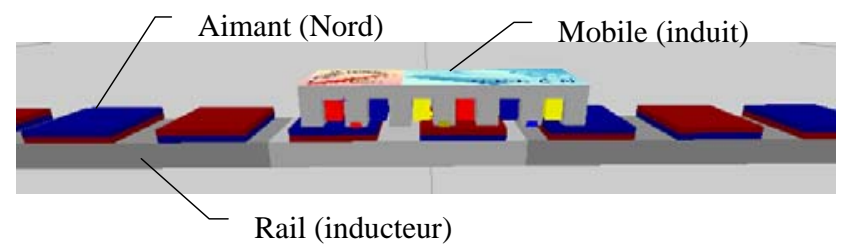

Fig 3. MSlin en simulation.

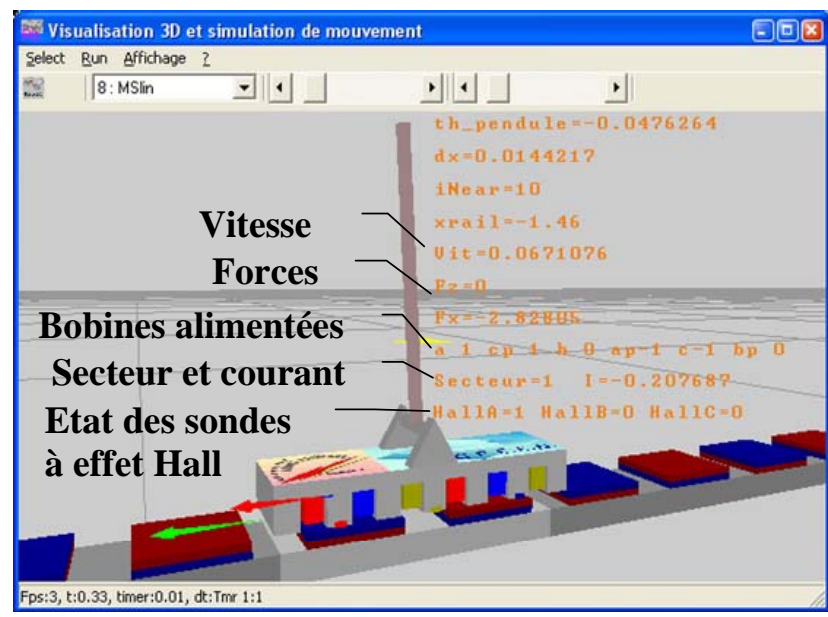

Fig 4. Simulation du MSlin avec pendule inverse.

\subsection{MRVlin}

C'est une machine $6 / 4$, les bobines sont donc au nombre de 6 ( 2 par phase, $\mathrm{p}=1$ ).

Elles sont alimentées de manière symétrique, 2 à 2 de manière à faire circuler le champ le long d'un parcours qui pousse à diminuer la réluctance du chemin et ainsi à faire déplacer le mobile plot par plot.

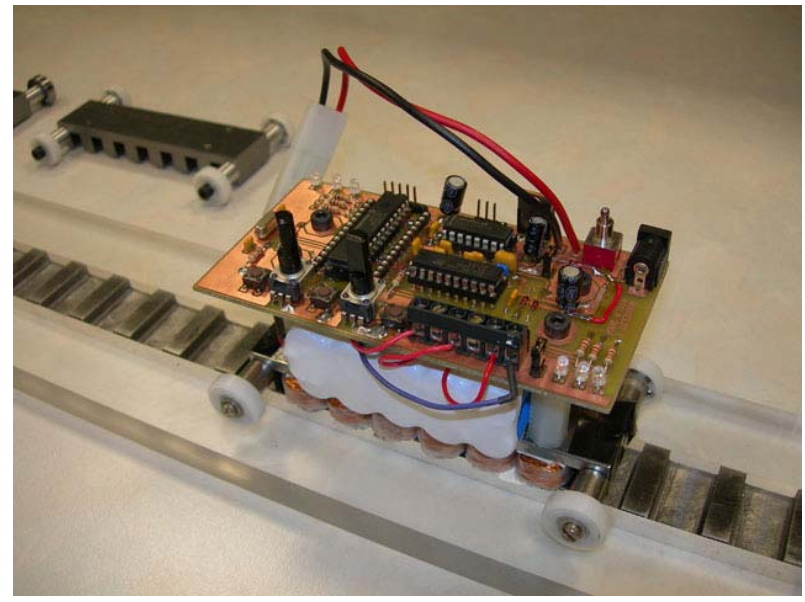

Fig 5. Photo de la version MRVlin avec batterie, on observe la carte de commande et d'électronique de puissance.

Si l'on prend des hypothèses simplificatrices : non saturation du matériau ferromagnétique, chute de force magnétomotrice dans l'acier nulle, énergie magnétique entièrement stockée dans le volume délimité par les deux surfaces des dents en regard (Fig 7), alors les équations électromagnétiques [3] donnent les résultats suivants :

$$
\begin{aligned}
& B=\frac{\mu_{0} 2 N i}{2 z}, \text { le volume } v=2 z\left(l_{x}-x\right) l_{y} \\
& W_{e}=\frac{B^{2}}{2 \mu_{0}} v=\mu_{0} N^{2} i^{2} \frac{\left(l_{x}-x\right) l_{y}}{z} \\
& F_{z}=-\frac{\partial W_{e}}{\partial z}=\mu_{0} N^{2} i^{2} \frac{\left(l_{x}-x\right) l_{y}}{z^{2}},
\end{aligned}
$$

Pour un entrefer $z=e=2 \mathrm{~mm}$ et des dents de dimensions $l_{x}=8 \mathrm{~mm}$ et $l_{y}=30 \mathrm{~mm}$,

$$
\begin{aligned}
& F_{z \max }=\mu_{0} N^{2} i^{2} \frac{l_{x} l_{y}}{e^{2}}=48 \mathrm{~N} \text { à } x=0 \mathrm{~mm} \\
& F_{x}=-\frac{\partial W_{e}}{\partial x}=\mu_{0} N^{2} i^{2} \frac{l_{y}}{z}, \\
& F_{x \max }=\mu_{0} N^{2} i^{2} \frac{l_{y}}{e}=6 \mathrm{~N}
\end{aligned}
$$

La force $F_{x}$ n'admet alors que 3 valeurs discrètes $\left(F_{x \max },-F_{x \max }\right.$ ou 0$)$ en fonction de $x$ :

$$
F_{x}= \begin{cases}F_{x \max } & x \in]-l_{x}, 0[ \\ -F_{x \max } & x \in] 0, l_{x}[ \\ 0 \text { ailleurs }\end{cases}
$$

Des calculs numériques montre qu'en réalité, la force de traction $F_{x}$ a plutôt le profil représenté par la Fig 6 .

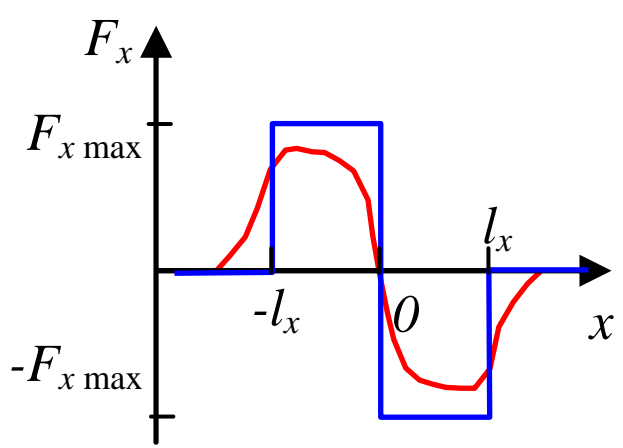

Fig 6. Profil de la force de traction en fonction de la position du mobile par rapport au rail. Modèle simple (en bleu),

calcul par la méthode des éléments finis (en rouge).

On observe que la force $F_{x}$ présente une grande discontinuité à $x=0$. Fort heureusement, l'amortissement dû au frottement permet un alignement des plots (après plusieurs oscillations) et à la stabilisation de cette position jusqu'au prochain changement de séquence d'alimentation (Fig 9, courbes bleues) [6]. 

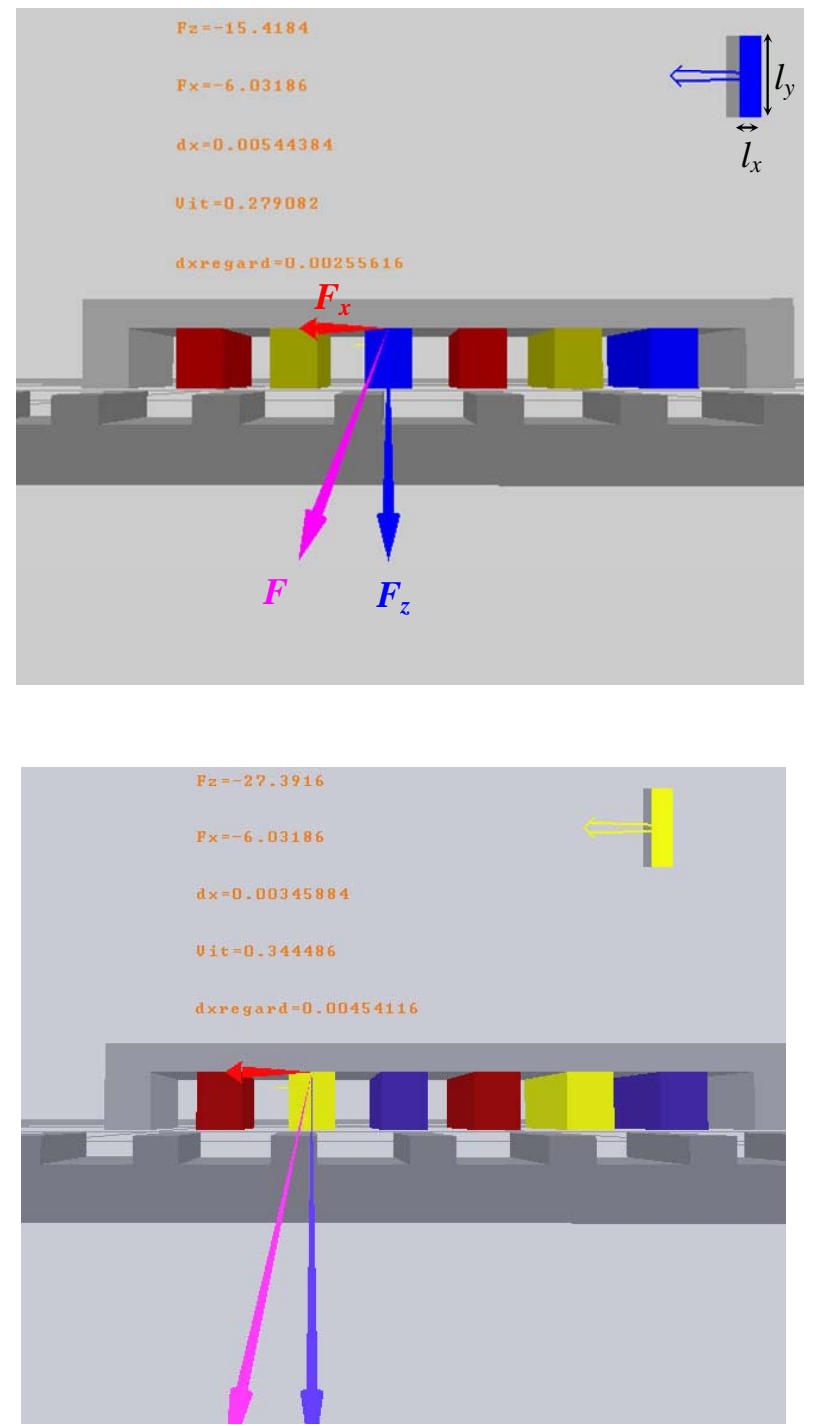

Fig 7. Configurations successives d'alimentation des bobines du MRVlin, visualisation à l'aide du logiciel de simulation.
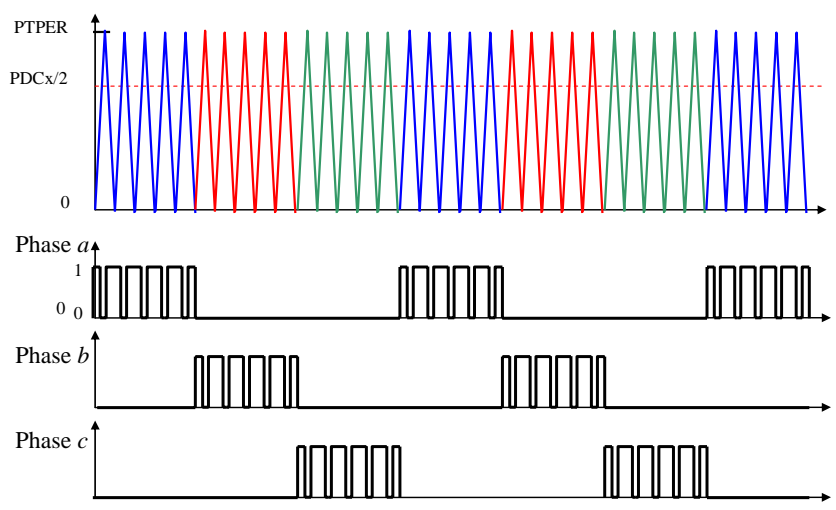

Fig 8. Séquence d'alimentation des phases, motifs de MLI
Les séquences d'alimentation sont appliquées par l'onduleur via la commande PWM du dsPIC. Ce dernier a un registre (OVDCON), qui permet d'inhiber ou non les sorties PWM indépendamment l'une de l'autre. Nous utilisons cette particularité pour faire défiler les séquences d'alimentation des bobines (Fig 8).

Deux potentiomètres sont fixés sur la carte de commande (Fig 12). Ils sont reliés aux entrées analogiques du dsPIC. Grâce au convertisseur analogique-numérique (10 bits) du dsPIC, les références de tension des potentiomètres sont converties en variables numériques (valeur de 0 à 1023) et permettent d'agir sur le comportement du mobile. Dans notre application, nous pouvons ainsi changer la fréquence de déroulement des séquences ainsi que le rapport cyclique de la PWM (et donc du niveau de courant dans les bobines).

Le mobile roule sur des rails en plexiglas mis de part et d'autre de la structure ferromagnétique du rail. Les roulements que nous utilisons servent à diminuer le frottement et à compenser la force $F_{z}$. Cette force de collage est très importante comparée à la force de traction $F_{x}$.

Nous voyons bien que cette manière de commander le MRVlin (en boucle ouverte) ne permet pas d'atteindre des vitesses de déplacement élevées car on ne peut pas augmenter la fréquence de déroulement des séquences d'alimentation sans altérer le mouvement correct du mobile.

En effet, si la séquence change alors que le mobile a entamé son oscillation autour du plot, il est freiné et peut même reculer.

L'idée est de commuter les séquences automatiquement dès que la dent du mobile se retrouve devant la dent correspondante du rail avant la première oscillation (Fig 9). On profite alors d'une vitesse positive pour aller encore plus vite vers la dent suivante. Ainsi, le mobile accélère avec une force constante $F_{x \max }$ (Fig 6). Un moyen simple que l'on a trouvé pour implanter cet autopilotage est d'utiliser un capteur optique (HOA 1405) qui émet un rayon infrarouge (IR) qui se reflète sur une surface plus ou moins sombre. Le flux lumineux est ensuite capté par le phototransistor IR, intégré au capteur, dont la focale est centrée sur un point à $5 \mathrm{~mm}$ en dessous du capteur. On place alors une série de traits noirs (Fig 10) sur une surface blanche (réfléchissante) espacé exactement de $l_{x}=8$ mm, c'est-à-dire, de l'écart entre deux plots du mobile. On réalise ainsi l'autopilotage comme le montre la vidéo [7]. Par le placement de la règle des traits noirs par rapport au rail ferromagnétique, on peut même régler "l'avance à l'allumage", à l'instar de ce qui se passe sur un moteur thermique.

Nous avons décliné la maquette en trois exemplaires : deux munis du capteur IR et un n'en disposant pas mais 
qui embarque une batterie d'accumulateurs $\mathrm{NiMH}$ fournissant $12 \mathrm{~V}$. Cela permet de faire quelques démonstrations en mode autoalimenté si on limite la valeur $V_{\text {ref }}$ et donc le courant dans la bobine.

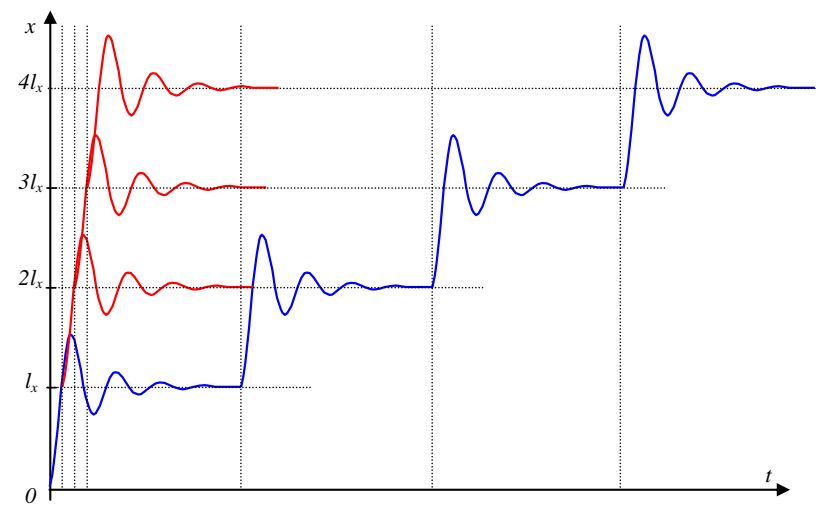

Fig 9. Oscillations autour des positions d'équilibre lors du déroulement des séquences : mode boucle ouverte (en bleu), autopilotage (en rouge).

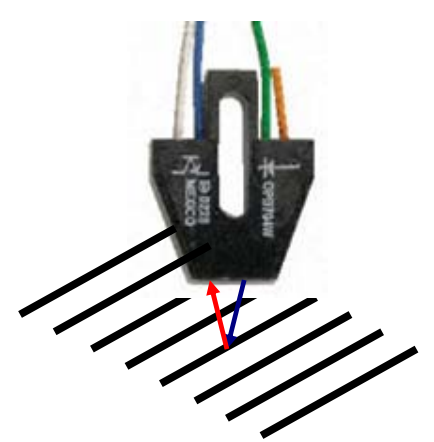

Fig 10. Lecture optique IR de la position du mobile par rapport aux dents du rail

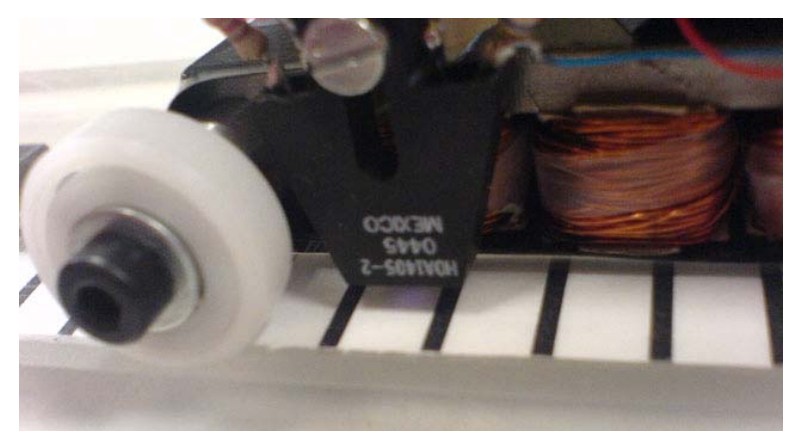

Fig 11. Agrandissement sur la partie du capteur IR, on observe le faisceau IR : tâche de couleur bleue mise en évidence par le CCD de l'APN.

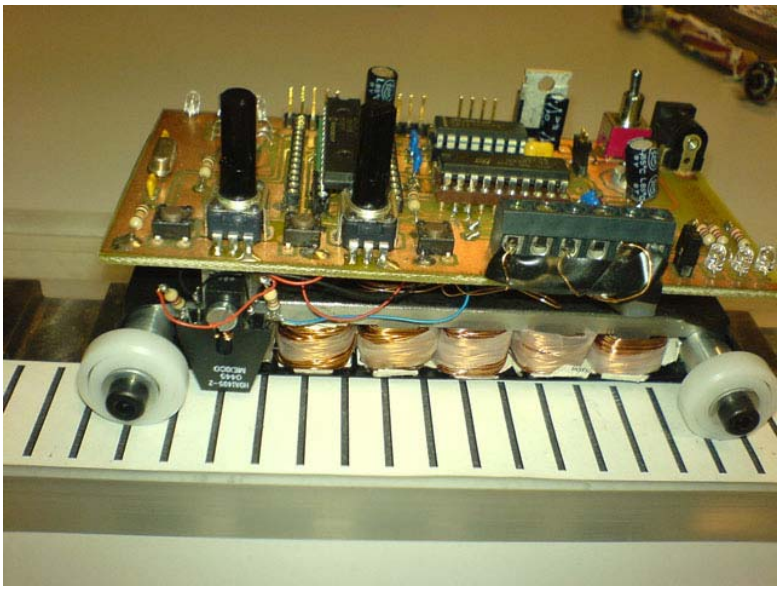

Fig 12. Photo de la version MRVlin sans batterie mais avec capteur IR.

\subsection{MSlin}

L'idée est de réaliser un moteur synchrone linéaire à aimants permanents. Il est constitué d'un mobile (induit) comportant un bobinage triphasé à une paire de pôles, une encoche par pôle et par phase et d'un rail en acier avec des aimants en NFeB plats collés à sa surface. Le mobile roule sur des rails en plexiglas attenant au rail ferromagnétique à aimants (Fig 16) [8],[9].

$\mathrm{Au}$ début du projet, nous avons construit le mobile dans le même matériau ferromagnétique que le rail mais on s'est aperçu (et vérifié à l'aide de FEMM [4]) que l'énorme force $F_{z}$ et surtout la force de détente en $x$ et des effets de bords du mobile (problème qui n'existe pas dans une machine ronde) provoquaient une force de collage nettement supérieure à la force de traction. Nous avons opté pour une solution simple qui consiste à fabriquer un mobile en acier inoxydable amagnétique. Cela a annulé toutes les forces de détente et la force $F_{z}$. Le contrôle du moteur est donc plus simple.

L'autopilotage de la machine ou commande 6 secteurs nécessite la connaissance de la position relative du bobinage par rapport aux aimants. Cela est souvent réalisé par 3 sondes Hall placées comme indiqué sur les Fig 13 et Fig 14.

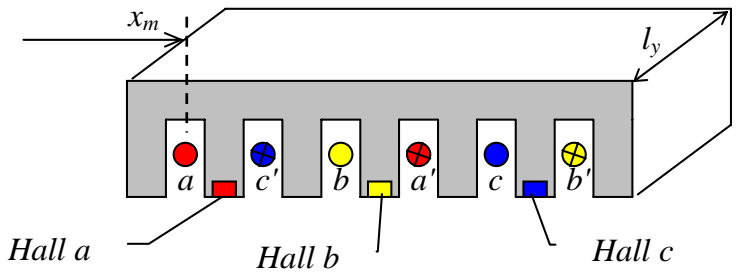

Fig 13. MSlin, bobinage et sondes Hall. 


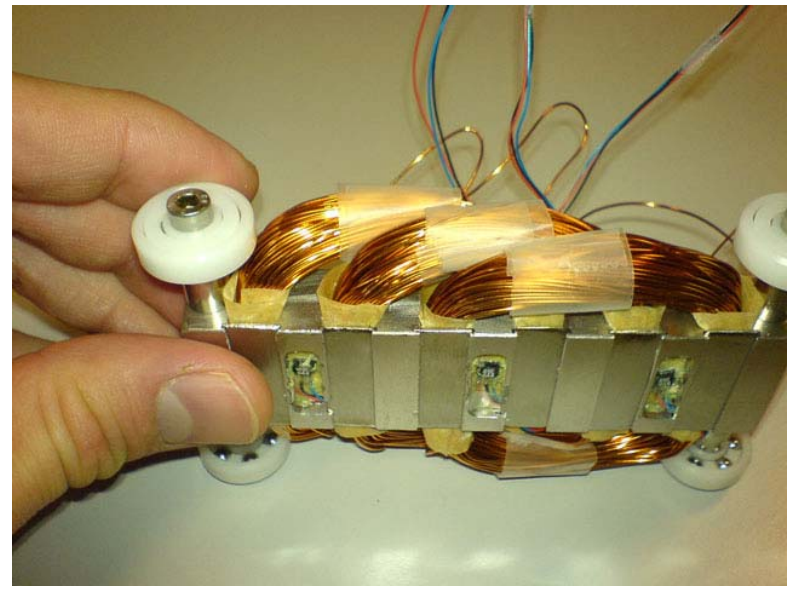

Fig 14. MSlin vu de côté, on observe les enroulements qui passent dans les encoches ( $\left.a, c^{\prime}, b, a^{\prime}, c, b^{\prime}\right)$ clavetés et les trois sondes Hall $(a, b, c)$.

Ces sondes vont voir l'induction des aimants $B$ et vont faire basculer leur sortie en conséquent, à $0 \mathrm{~V}$ ou $+5 \mathrm{~V}$, selon qu'elles soient au dessus d'un Sud ou d'un Nord (Fig 15).

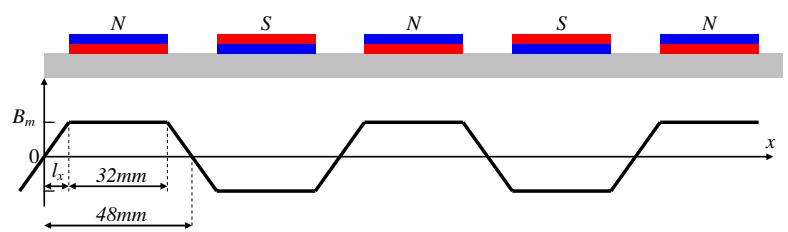

Fig 15. Induction générée par les aimants le long du rail.

On génère alors numériquement dans le programme de commande du dsPIC, une variable appelé Secteur :

$$
\text { Secteur }=2^{0} \cdot \text { Hall } a+2^{1} \cdot \text { Hall } b+2^{2} \cdot \text { Hall } c
$$

Cette variable va prendre les valeurs $(3,2,6,4,5,1)$ successivement quand le mobile se déplace.

On impose des courants dans les bobines de sorte que si les faisceaux $a$ et $c^{\prime}$ des bobines sont face à un Nord, que les faisceaux b et b' sont face à une pente de $B$ croissant ou décroissant et que les faisceaux $a^{\prime}$ et $c$ sont face à un Sud, alors on doit alimenter les phases respectivement par $+I, 0$ et $-I$.

On obtient alors 4 forces (loi de Laplace) qui vont s'additionner pour agir sur le mobile.

$F_{x}=4 F_{a}=4 I N l_{y} B_{\mathrm{m}}=6.7 \mathrm{~N}$

Quand le mobile se déplace, le calcul du secteur se refait et une nouvelle configuration est générée. La caractéristique de ce contrôle est que seuls deux enroulements sont alimentés simultanément.

Nous avons alors imposé un rapport cyclique de 50\% sur la phase qui doit être débranchée, afin d'y faire circuler un courant nul sur une période MLI.

Exemple du secteur 1, la configuration des registres des comparateurs du module PWM du dsPIC est :
PDC1 = Vref;

PDC2 = HalfDUTY;

PDC3 = FullDUTY-Vref;

Afin de pouvoir montrer le fonctionnement de l'autopilotage, nous avons doté le MSlin d'un écran LCD 2x16 caractères, piloté par liaison série SPI (voir programme source [8]). Nous y affichons l'état des 3 sondes Hall, le secteur actuel et la configuration d'alimentation des bobines. En déplaçant lentement le mobile sur les rails au dessus des aimants, les étudiants pourront observer le déroulement des séquences et des configurations [9].

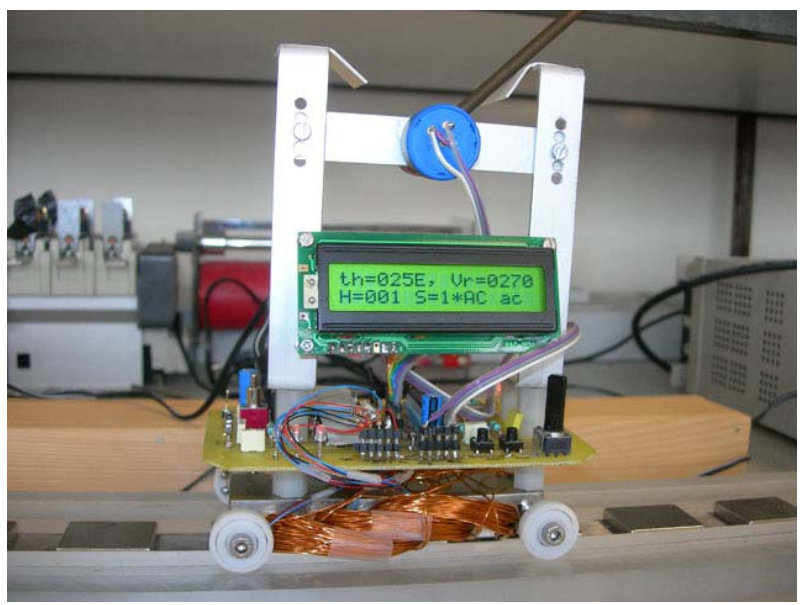

Fig 16. MSlin, visualisation de l'état des sondes Hall, du secteur actuel et de la configuration d'alimentation des bobines.

Afin de montrer la dynamique qu'offre cet actionneur, nous avons rajouté un pendule inverse dont on va réguler la position à la verticale.

Le pendule inverse étant de nature instable, il a fallut utiliser un régulateur Proportionnel-Dérivé (PD) au lieu du traditionnel Proportionnel-Intégral (PI).

Un potentiomètre à recouvrement permet de donner l'angle du pendule. Cet angle est affiché sur l'écran LCD en temps réel.

Tant que le MSlin dispose d'espace le long du rail, il essaye de réguler l'angle du pendule, rejetant les perturbations, comme celles que lui communique l'utilisateur en donnant des à-coups [10].

Nous avons rajouté un potentiomètre qui permet de limiter la tension maximale en sortie du régulateur PD du pendule inverse et une protection qui inhibe l'onduleur si l'angle du pendule devient très important (quand le pendule bloque sur une des deux butés).

Nous avons été confrontés, lors des tests de la maquette à un problème de CEM. Le dsPIC fonctionnait correctement quand de faibles courants étaient appliqués au bobinage, mais dès que ces courants augmentaient, cela provoquait une remise à zéro (reset) du dsPIC. Après quelques investigations, 
nous nous sommes aperçus que cela était dû à un problème de retour de masse. Par conséquent, nous avons séparé les retours des courants de l'onduleur et du circuit numérique et nous les avons connectés en un seul point, à la masse de l'alimentation. Rappelons que pour ces deux moteurs linéaires, l'électronique de commande est alimentée sous $5 \mathrm{~V}$ à partir d'un régulateur 7805 qui puise son énergie de l'alimentation générale (12V pour le MRVlin et $9 \mathrm{~V}$ pour le MSlin).

\subsection{MASlin}

Pourquoi la MASlin n'a pas "marché" ? En réalité, la même structure du mobile peut être utilisée pour réaliser un champ glissant. Il suffit d'une routine qui implémente une table de sinus de 256 valeurs numérisées et d'une transformation de Park adéquate. Les tensions de références ainsi obtenues sont transcrites en rapports cycliques de MLI.

Cependant, les courants et les tensions mis en jeu ne permettaient pas d'induire suffisamment de courant dans le rail pour produire le champ suffisant (équivalent du champ rotorique d'une MAS classique) et donc une force permettant la mise en mouvement de la machine.

Il faudrait augmenter le nombre de spires et le niveau de tension de manière à avoir des fem plus importantes et apporter plus d'énergie réactive au rail (équivalent du rotor). Evidement, ce problème ne se pose pas pour la MSlin puisque elle dispose d'un rail muni d'aimants.

\section{CONCLUSION}

L'expérience menée ici est encore nouvelle, nous avons utilisé le MRVlin l'année dernière et le MSlin cette année. Nous savons d'ores et déjà qu'il y a un apport positif ne serait ce que du point de vue de l'engouement et de la motivation à comprendre les phénomènes mis en jeu pour animer ces dispositifs. En effet, les étudiants sont agréablement surpris de les voir bouger et de réaliser que les équations et développement théoriques du cours et des travaux dirigés qu'ils trouvent si difficiles et muets, prennent vie dans des petits actionneurs tangibles.

L'autre intérêt, de cette approche projet, est que l'on peut approfondir plusieurs thématiques connexes : Electromagnétisme (Laplace, énergie / coénergie, Tenseur de Maxwell), Electronique de puissance (hacheur, onduleur triphasé), Automatique (modélisation, régulation, systèmes échantillonnés, discrétisation, temps réel), Informatique (programmation, $\mathrm{C}++$, OpenGL, API Windows, analyse numérique, RK/Euler), Informatique industrielle (microcontrôleur, programmation temps réel, ISR, ADC, PWM, liaison série asynchrone et synchrone (SPI), LCD, interface homme-machine), Gestion de projet...

Nous espérons que ce projet, diffusé sur internet et à travers cet article, se trouvera copié et dupliqué. Nous serions heureux d'avoir des retours et d'éventuelles améliorations aux machines présentées.

\section{Remerciements}

Les auteurs tiennent à remercier les personnes suivantes pour la réalisation des pièces mécaniques et des cartes électroniques :

C. Busselot, J.F. Pautex, ATELA - UHP.

J.Y. Morel, F. Sharif, M. Thiebaut, ENSEM - INPL.

\section{Bibliographie}

1. L. Baghli, "Projets autour du dsPIC", [En ligne]. Disponible: http://www.baghli.com/dspic.php

2. L. Baghli, "Simulation of different kind of Robots in a 3D visualisation scene under C++ BDS 2006 and OpenGL", [En ligne]. Disponible : http://www.baghli.com/bds_robots.html\#MRVlin 3. M. Juffer, Traité d'électricité vol IX. Electromécanique, Ed. PPURomandes, 3ème édition, 1995, 384p.

4. D. C. Meeker, Finite Element Method Magnetics, Version 4.0.1 (03Dec2006 Build), [En ligne].

Disponible:http://femm.foster-miller.net

5. L. Baghli, "MRVlin, le projet", [En ligne].

Disponible: http://www.baghli.com/dspic.php\#MRVlin

6. L. Baghli, "MRVlin, vidéo 1, marche en boucle ouverte", [En ligne]. Disponible:

http://www.youtube.com/watch?v=w_Kera5NTQM

7. L. Baghli, "MRVlin, vidéo 2, autopilotage", [En ligne]. Disponible:

http://www.youtube.com/watch?v=zDWH5hmTmi8

8. L. Baghli, "MSlin, le projet", [En ligne].

Disponible: http://www.baghli.com/dspic.php\#MSlin

9. L. Baghli, "MSlin, vidéo 1, explication", [En ligne]. Disponible:

http://www.youtube.com/watch?v=wsc-ypGp9qE 10. L. Baghli, "MSlin, vidéo 2, régulation du pendule inverse", [En ligne]. Disponible:

http://www.youtube.com/watch?v=DWm-DAZGlKA 